\title{
Factores de Riesgo para la Diabetes Mellitus en el Profesional de Enfermería
}

\section{Risk Factors for Diabetes Mellitus in Nursing Professional}

Mtro. Francisco Javier Báez Hernández,• Mtra. Marcela Flores Merlo,••

Est. Pregrado Elizabeth Bautista Barranco,..• Est. Pregrado Liliana Sánchez Hernández...•

\section{Resumen}

Introducción: los factores de riesgo para la Diabetes Mellitus, son aquellos que aumentan la posibilidad de padecer la enfermedad. Su conocimiento permite ser la base para el desarrollo de actividades preventivas y promotoras de salud. Objetivo: identificar los factores de riesgo para la Diabetes Mellitus en un grupo de enfermeras, mediante los conceptos de la Norma Oficial Mexicana 015 y lo propuesto por la OMS. Metodología: el estudio fue de tipo descriptivo, transversal, se realizó en un grupo de profesionales de Enfermería inscritas en un programa de nivelación académica. A través de un muestreo no probabilístico por conveniencia. La información se obtuvo mediante una cédula de datos personales y el Cuestionario de Factores de Riesgo de la Secretaría de Salud. Resultados: La media para el Cuestionario de Factores de Riesgo para la Diabetes Mellitus de 11.85 (DE=9.06), lo que significa la posibilidad de padecer la enfermedad en el profesional de Enfermería. Discusión y Conclusiones: los hallazgos en este estudio ubican a la población en un riesgo alto para desarrollar Diabetes Mellitus, resultados que coinciden con los obtenidos en otros estudios.

\section{Abstract}

Introduction: risk factors for diabetes mellitus are those that increase the chance of developing the disease. His knowledge, allows it to be the basis for the development of preventive and health promotion. Objective: To identify risk factors for diabetes mellitus in group of nurses, using the concepts of the Norma Official Mexicana 015 and proposed by the WHO. Methodology: The study was descriptive, transversal, was conducted in a group of nursing professionals enrolled in an academic program placement. Through a non-probability convenience sampling. The information was obtained through a certificate of personal data and the Risk Factor Questionnaire of the Ministry of Health. Results: We found an average for the Survey of Risk Factors for Diabetes Mellitus of 11.85 (SD = 9.06), which means the chance of developing the disease in the nursing professional. Discussion and Conclusions: The findings in this study placed the population at high risk for developing diabetes mellitus. Results that agree with those obtained in other studies.

- Maestro en Ciencias de Enfermería, Profesor Investigador de la Benemérita Universidad Autónoma de Puebla. •-Maestra en Ciencias de Enfermería, Profesora Investigadora de la Benemérita Universidad Autónoma de Puebla. ...Estudiantes de Pregrado en Enfermería de la Benemérita Universidad Autónoma de Puebla.

Correspodencia: jav007@hotmail.com

RECIBIDO 8 DE ENERO 2011

ENVIADO 11 DE ENERO 2011

ACEPTADO 4 DE FEBRERO 2011 


\section{INTRODUCCIÓN}

La Diabetes Mellitus (DM) es definida como un grupo de enfermedades metabólicas caracterizadas por hiperglucemia, a consecuencia de defectos en la secreción y acción de la insulina. ${ }^{1}$

En América Latina y el Caribe, el número de personas que padecen esta enfermedad se estima en 19 millones, cantidad que podría incrementarse a 40 millones en 2025, si no se introducen acciones

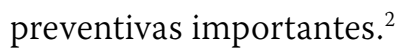

En México la prevalencia de personas con la enfermedad aumentó a $14 \%$, lo que representa un total de 8 millones de individuos. La DM es la primera causa de incremento para las estadísticas de morbilidad y de mortalidad, puesto que origina: $12 \%$ de los casos nuevos de ceguera, 25\% de los casos de insuficiencia renal, $40 \%$ de todas las amputaciones no traumáticas de miembros pélvicos en adultos. En el estado de Puebla la tasa de Prevalencia de Diabetes en adultos de 20 años o más es de $6.3 \%$, siendo mayor en hombres que en mujeres (6.9\% vs $5.8 \%) .^{3}$

La DM es considerada una enfermedad crónica y a la vez un factor de riesgo para desarrollar enfermedad cardiovascular, hipertensión arterial y accidente vascular cerebral. ${ }^{4}$

Según la Norma Oficial Mexicana 015 (NOM-015) y la Organización Mundial de la Salud (OMS), los factores de riesgo para la DM, son aquellos que aumentan la posibilidad para el desarrollo de la enfermedad, principalmente: antecedentes hereditarios (que un hermano, padre o madre consanguíneo padezcan DM), edad mayor de 45 años (dado que cronológicamente se tiene mayor probabilidad de padecer DM tipo dos), falta de actividad física (conocida como sedentarismo o caminar menos de media hora todos los días), ${ }^{5}$ sobrepeso y obesidad, definidos como un índice de masa corporal (IMC) mayor a 25 y 30 respectivamente y una circunferencia de cintura (CC) mayor de $80 \mathrm{~cm}$ para mujeres y 90 $\mathrm{cm}$ en hombres, dado que se correlaciona con el acúmulo de grasa en órganos no grasos como corazón, hígado, músculo, riñón y páncreas, situación que conlleva a un proceso de resistencia a la insulina con hiperinsulinemia compensatoria que finalmente se manifiesta con glicemias mayores a $130 \mathrm{mg} / \mathrm{dl}) \cdot{ }^{6,7} \mathrm{En}$ mujeres la $(\mathrm{CC})>80 \mathrm{~cm}$ se correlaciona con haber padecido diabetes gestacional o haber tenido productos macrosómicos (mayor a $4 \mathrm{Kg}$.). ${ }^{8}$

Otros factores de riesgo para el desarrollo de DM, no menos importantes son; estrés prologado, dado que se ha vinculado este con ciertas sustancias o moléculas elementales para el metabolismo de glucosa; ${ }^{9}$ consumo de alcohol (de más de dos copas al día en hombres y más de una en mujeres) $)^{10,11}$ y tabaco, ${ }^{12}$ así como de medicamentos como las tiazidas, glucocorticoides, difenilhidantoina y bloqueadores beta-adrenérgicos. ${ }^{13}$

Considerando que el profesional de enfermería es uno de los principales elementos del equipo de salud para disminuir la prevalencia de esta enfermedad y sus complicaciones, utilizando estrategias clínicas y educativas que ayudan a mantener la salud de los individuos. ${ }^{14,16}$ Se hace evidente la importancia de identificar dichos factores de riesgo que pudieran presentarse en éste colectivo para padecer DM.

\section{Objetivo}

Identificar los factores de riesgo para DM en el profesional de Enfermería, mediante los conceptos de la Norma Oficial Mexicana 015 y lo propuesto por la OMS.

\section{Metodología}

El presente estudio fue de tipo descriptivo, transversal ${ }^{17}$ Se realizó en un grupo de profesionales de Enfermería inscritas en un programa de nivelación académica, a través de un muestreo no probabilístico por conveniencia. La muestra fue calculada mediante el programa estadístico STATS-TM, versión 2.0, con un tamaño del universo de 300 , un margen aceptable de error del $4 \%$, una muestra estimada de $50 \%$ y un intervalo de confianza del 95\%, obteniendo una $\mathrm{n}=150$.

Los criterios de exclusión fueron: padecer la enfermedad y estar embarazada.

La información se obtuvo mediante una cédula de datos generales y el cuestionario de Factores de Riesgo para la Diabetes Mellitus de la Secretaría de Salud. La cédula de datos personales se compone de 13 preguntas que abordaron las siguientes variables: edad, género, antecedentes heredofamiliares de DM, tipo de diabetes, sobrepeso $u$ obesidad, consumo de alcohol y/o tabaco, frecuencia de actividad física e ingesta de medicamentos derivados de las tiazidas, glucocorticoides, difenilhidantoina y bloqueadores beta-adrenérgicos.

El Cuestionario de Factores de Riesgo para la Diabetes Mellitus de la Secretaría de Salud está formado por 8 preguntas, con puntuación mínima y máxima de todo el instrumento, de 0 a 42 puntos; con dos opciones de respuesta: sí y no. 
La pregunta uno ubica a la persona de acuerdo a su Índice de IMC en: normal, sobrepeso y obesidad. La opción de respuesta no, equivale a normal con valor de 0 , la opción sí corresponde a: sobrepeso con un valor de 5 y a la obesidad con un valor de 10. La pregunta dos se refieren a la circunferencia de cintura, reciben 10 puntos aquellos que obtienen valores por arriba de lo normal y cero para lo que no.

La pregunta tres, cuatro y cinco la puntación de la opción sí, corresponde a 5 , y hacen referencia a la actividad física y a la edad de la persona. Las preguntas 6,7 y 8 la opción de respuesta sí, equivalen a un punto, y corresponden a cuestionamientos acerca de los antecedentes heredofamiliares para la DM. Los individuos que obtienen una calificación menor de 10 puntos, tienen un menor riesgo de desarrollar la enfermedad. Las personas que obtienen una calificación igual o mayor de 10, tienen un riesgo alto para padecer Diabetes Mellitus. ${ }^{18}$

Para la medición de peso y talla se solicitó a las participantes acudir con ropa ligera y en ayuno. En una báscula se obtuvieron los datos para clasificar el IMC de acuerdo a lo establecido por la OMS: Un puntaje de 18.5 a 24.9 como Normal, de 25.0 a 29.9 Sobrepeso, 30.0 a 34.9 Obesidad tipo I, 35.0 a 39.9 Obesidad tipo II y mayor o igual a 40 Obesidad tipo III. ${ }^{19}$ También se realizó la medición de la Circunferencia de Cintura (CC), la técnica se realizó de acuerdo a lo señalado en Internacional Society for the Advancement of Kinanthropometry (ISAK), ${ }^{20}$ ajustando la cinta en la parte más angosta de la cintura sin comprimir la piel, manteniendo la cinta en un plano horizontal, paralelo al piso. Se tomaron dos mediciones, si la diferencia fue mayor a $2.5 \mathrm{~cm}$ se midió una vez más para disminuir la variabilidad. El registro de la CC se realizó al final de la exhalación redondeando la medición al siguiente medio centímetro.

Para realizar la presente investigación, se solicitó la autorización del Comité de Ética de la Facultad de Enfermería de la Benemérita Universidad Autónoma de Puebla (FE-BUAP), así como al Departamento de Nivelación Académica (DeNa).

Para la recolección de los datos se comenzó con tomar la somatometría en uno de los laboratorios clínicos de la FE-BUAP, por un solo integrante del grupo de investigadores con el propósito de disminuir la variabilidad en las técnicas antropométricas. Posteriormente se les invitó a contestar el Cuestionario de Factores de Riesgo para la Diabetes Mellitus de la Secretaria de Salud en el Auditorio de la Facultad, previo consentimiento informado y autorización oral y escrita de los participantes. Las encuestas fueron depositadas en una urna especial, con la finalidad

\section{Tabla 1. Características Personales del Profesional de Enfermería.}

\begin{tabular}{llrr}
\hline \multirow{2}{*}{ Variable } & Caracteristicas & Fx & \% \\
\hline Género & Femenino & 143 & 95.3 \\
& Masculino & 7 & 4.7 \\
& Total & 150 & 100 \\
& & & \\
Lugar de Trabajo & Primer Nivel & 39 & 26.0 \\
& Segundo Nivel & 58 & 38.7 \\
& Tercer Nivel & 53 & 35.3 \\
& Total & 150 & 100
\end{tabular}

$n=150$ de asegurar la confidencialidad de los encuestados. Al finalizar se les agradeció su participación.

Se tomó en consideración lo dispuesto en la Ley General de Salud del 2000, de acuerdo a los artículos 13, 14 y 16 que especifican los principios para la investigación con seres humanos. ${ }^{21} \mathrm{El}$ análisis de los datos se realizó mediante el paquete estadístico Statistical Package For the Social Sciences (SPSS) versión 12 , mediante el uso de estadística descriptiva como tablas de frecuencias, porcentajes, medidas de tendencia central y dispersión.

\section{Resultados}

La muestra quedó constituida por 150 enfermeras y la media de edad fue 34.21 ( $D E=7.23$ ), con un rango de 25 a 51 años. En cuanto al género de los participantes se encontró que el 95.3\% (143) fueron mujeres y el 4.7\% (7) hombres.

En relación al lugar laboral, se obtuvo un $38.7 \%$ (58) para el segundo nivel de atención, seguido del tercer nivel (35.3\%), (53) y el primer nivel de atención de salud (26.0\%), (39) (ver tabla 1).

Para el apartado de características heredofamiliares del profe- 
sional de Enfermería, se encontró que el 13.3\% (20), tiene algún hermano con Diabetes Mellitus; 4.7\% (7) del tipo I y $8.0 \%$ (13) del tipo II. En cuanto a la existencia del padecimiento en alguno de sus padres, se encontró que el 42.7\% (64) tienen Diabetes Mellitus; 10.0\% (15) del tipo I y $32.7 \%$ (49) del tipo II. En relación de haber obtenido un producto con peso al nacer mayor de 4kgs., sólo el 5.3\% refirió haber concebido un hijo con esas características (ver tabla 2).

La media del puntaje obtenida del Cuestionario de Factores de Riesgo para la Diabetes Mellitus fue 11.85 ( $\mathrm{DE}=9.06)$, lo que significa un riesgo alto de padecer la enfermedad.

Las medias obtenidas en el IMC $(X=25.47 ; D E=3.76)$ y de Circunferencia de Cintura ( $\mathrm{X}=84.64$; $\mathrm{DE}=9.74$ ), ubica a la población de estudio en sobrepeso y en un riesgo incrementado para desarrollar complicaciones metabólicas relacionadas con la obesidad (ver tabla 3 ).

En relación al nivel de riesgo se encontró que el 56.7\% (85) tienen un alto riesgo de padecer DM, (ver tabla 4).

En cuanto a la clasificación por IMC, se obtuvo que el $47.3 \%$ correspondió a normal y el $52.7 \%$ tuvo algún grado de sobrepeso y obesidad (ver tabla 5). De acuerdo a lo obtenido en la circunferencia de cintura se encontró que el 41.3\% (62), tiene un riesgo sustancialmente incrementado, seguido del $30.7 \%$ (46) con un riesgo incrementado (ver tabla 6).

En referencia a la actividad física se reportó que el $40.7 \%$ (61) no camina al menos media hora todos los días. En relación al consumo de alcohol y tabaco solo el 18\% (27) y

\section{Tabla 2. Características Heredofamiliares del Profesional de Enfermería.}

\begin{tabular}{|l|rrr}
\hline Variable & Características & Fx & $\%$ \\
\hline Hermanos con Diabetes Mellitus & Si & 20 & 13.3 \\
& No & 130 & 86.7 \\
& Total & 150 & 100 \\
& & & \\
\hline \multirow{2}{*}{ Padres con Diabetes Mellitus } & Si & 64 & 42.7 \\
& No & 86 & 57.3 \\
& Total & 150 & 100 \\
\hline Hijos con Peso al Nacer Mayor de 4 Kg. & Si & & \\
& No & 8 & 5.6 \\
& Total & 135 & 94.4 \\
& & 143 & 100 \\
\hline * Mujeres & & & \\
$\mathrm{n}=150$ & & &
\end{tabular}

\section{Tabla 3.Variables de Riesgo para la Diabetes Mellitus en Profesionales de Enfermería.}

\begin{tabular}{lccrr}
\hline Variable & X & DE & Valor Min & Valor Max \\
\hline Factor de Riesgo & 11.85 & 9.06 & 0 & 32 \\
Índice de Masa Corporal & 25.47 & 3.76 & 18.50 & 38.09 \\
Circunferencia Cintura & 84.64 & 9.74 & 60 & 115 \\
\hline n=150
\end{tabular}

Tabla 4. Nivel de Riesgo para la Diabetes Mellitus en Profesionales de Enfermería.

\begin{tabular}{|c|c|c|c|}
\hline Variable & Característica & $F x$ & $\%$ \\
\hline \multirow{3}{*}{ Nivel de riesgo } & Con Bajo Riesgo & 55 & 43.4 \\
\hline & Con Alto Riesgo & 85 & 56.7 \\
\hline & Total & 150 & 100 \\
\hline
\end{tabular}

\section{Tabla 5. Clasificación de IMC en Profesionales de Enfermería}

\begin{tabular}{|c|c|c|c|}
\hline Variable & Característica & $F x$ & $\%$ \\
\hline \multirow[t]{5}{*}{ IMC } & Normal & 71 & 47.3 \\
\hline & Sobrepeso & 58 & 38.7 \\
\hline & Obesidad Tipo I & 18 & 12.0 \\
\hline & Obesidad Tipo II & 3 & 2.0 \\
\hline & Total & 150 & 100 \\
\hline
\end{tabular}




\section{Tabla 6. Tipo de Riesgo para Desarrollar Complicaciones Metabólicas Relacionadas con la Obesidad en Profesionales de Enfermería.}

\begin{tabular}{llrr} 
Variable & Característica & Fx & $\%$ \\
\hline Circunferencia Cintura & Sin Riesgo & 42 & 28.0 \\
& Con Riesgo Incrementado & 46 & 30.7 \\
& Con Riesgo Sustancialmente Incrementado & 62 & 41.3 \\
& Total & 150 & 100 \\
\hline $\mathrm{n}=150$ & &
\end{tabular}

el 10.7\% (16) refirió ingerir alcohol y fumar cigarrillos.

\section{Discusión}

En el presente estudio se identificaron los factores de riesgo para padecer DM, mediante los conceptos de la Norma Oficial Mexicana 015 y lo propuesto por la OMS.

Se encontró que cerca de la mitad de la muestra estudiada refirió que sus padres padecen DM. Hechos que concuerdan con lo obtenido por Arenas y Cols, ${ }^{22}$ al mencionar en su estudio que los factores de riesgo más comunes son los antecedentes hereditarios, al igual que Bañuelos y Cols, ${ }^{23}$ en poblaciones de mujeres universitarias y habitantes de colonias del municipio de Durango.

Para los resultados de IMC y de $\mathrm{CC}$, se encontró un porcentaje alto de sobrepeso y riesgo incrementado para desarrollar complicaciones metabólicas relacionadas con la obesidad, resultados que coinciden con los obtenidos por Arenas, ${ }^{22}$ en un grupo de 37 universitarias, al identificar al sobrepeso y a la obesidad, como principales factores personales para el desarrollo de enfermedades crónicas no transmisibles. Resultados que pudiera deberse a un estilo de vida similar entre estos dos grupos.

También se encontró en relación a la actividad física, un alto porcentaje de profesionales de Enfermería que no realizan ejercicio. Datos que son semejantes a lo obtenido por $\mathrm{Hu}$, Manson, y Col, ${ }^{24}$ al encontrar en su investigación con profesionales de enfermería, como principal factor de riesgo para la DM, la falta de actividad física. Lo que pudiera reafirmar lo mencionado por Varo, Martínez y Martínez, ${ }^{25}$ en su estudio sobre los beneficios de la actividad física y riesgos del sedentarismo, en el que si bien, los datos de estos estudios no son directamente extrapolables a la población general, dado que sus participantes son todos profesionales sanitarios, es lógico que se piense que el impacto podría ser aún más llamativo si se consiguiese en la población general (más sedentaria) la adopción de un estilo de vida físicamente activo.

Por otra parte, para los resultados del consumo de Alcohol y Tabaco se coincide con lo obtenido por Mozaffarian, y Col, ${ }^{26}$ en su estudio sobre el estilo de vida, como factor predictor para padecer DM, en un grupo de adultos mayores, al obtener un bajo porcentaje para estas adiciones. Situación que pudiera deberse a un estilo de vida similar entre estas dos muestras.

\section{Conclusiones}

Los hallazgos en este estudio ubican a la población estudiada en un riesgo alto para desarrollar Diabetes Mellitus, así como complicaciones metabólicas relacionadas con la obesidad.

\section{Agradecimiento}

Al Dr. Erick Landeros Olvera por su asesoría, así como al profesional de Enfermería que participó en esta investigación.

\section{Referencias Bibliográficas}

1. American Diabetes Association: Diagnosis and Classification of Diabetes Mellitus. Diabetes Care 2006; 29 (Suppl 1): S43-S48.

2. Organización Panamericana de la Salud. Iniciativa de Diabetes para las Américas: Plan de Acción para América latina y el Caribe 20012006. (Consultado 09/09/2010). Disponible en: http://www.paho. org/spanish/AD/DPC/NC/D.

3. Olaiz G, Rivera J, Shamah T, Rojas R, Villalpando S, Hernández M, et al. Encuesta Nacional de Salud y Nutrición. Cuernavaca, México: Instituto Nacional de Salud Pública, 2006.

4. National Institute of Diabetes and Digestive and Kidney Diseases. The effect of intensive treatment of diabetes on the development and progression in insulin-dependent Diabetes Mellitus. N Engl J Med 1993; 329:977-86.

5. Van SE, Van PM, Twisk JB, \& Van MW. The positive effect on deter- 
minants of physical activity of tailored, general practice-based physical activity intervention. Health Education Research. 2005; 20(3): 345-56.

6. Bastarrachea R, Fuenmayor R, Brajkovich I, \& Comuzzie A. Entendiendo las causas de la obesidad a través de la biología celular del adipocito. Revista Venezolana de Endocrinología Metabólica. 2005; 3(3): 20-9.

7. Bastarrachea R, López AJ, Bolado V, Téllez M, Laviada M, \& Comuzzie A. Macrófagos, inflamación, tejido adiposo, obesidad y resistencia a la insulina. Gaceta Médica Mexicana. 2007; 143(6): 505-12.

8. Secretaría de Salud. Norma Oficial Mexicana 015 (1994). Para la Prevención, Tratamiento y Control de la Diabetes Mellitus en la Atención Primaria.

9. Bastarrachea R, Curran J, Bolado V, Kent J, López AJ, Téllez MJ, et al. Vinculando la respuesta inflamatoria, la obesidad y la diabetes con la sobrecarga (estrés) del retículo endoplasmático a través de las acciones de la seleno proteína S. Revista de Endocrinología y Nutrición. 2006; 14(2): 89-101.

10. Gunzerath L, Faden V, Zakhari S, Warren K. National Institute on Alcohol Abuse and Alcoholism Report on Moderate Drinking. Alcoholism: Clinical and Experimental Research, 2004, 6(28):829-47.

11. Feillin DA, Reid MC, O'Connor PG. Screening for alcohol problems in primary care. A systematic review. Arch Intern Med.2000; 160: 1977-89.

12. Will C, Bodenmann P, Ghali PD, Faris PD, Cornuz J. Active Smoking and the Risk of Type 2 Diabetes. A Systematic Review and Meta-analysis. The Journal of the
American Medical Association. 2007; 22 (298): 2654-64.

13. Instituto Nacional de Salud Pública. Diabetes Mellitus. Boletín de Práctica Médica. 2006,1-10.

14. Báez FJ, Hernández J, Marín MA, Villegas S. Intervención Educativa de Enfermería a persona con Diabetes Mellitus. Desarrollo Científico de Enfermería. 2009, 17(6): 252-5.

15. Gallegos E, Bañuelos Y. Conductas Protectoras de Salud en Adultos con Diabetes Mellitus Tipo II. Investigación y Educación en Enfermería. 2004. 23(2): 40-9.

16. Ontiveros S, Flores P, Castro A. Conocimiento de Enfermería en el Cuidado de Pacientes Diabéticos. Enfermería Global. 2007; 11; 1-9.

17. Polit, Huntler. Investigación Científica en Ciencias de la Salud. 6ta ed. México D.F: McGraw Hill Interamericana, 2000.

18. Secretaría de Salud. Dirección General de Información de Salud. [Recuperado el 13 de Septiembre de 2007]. Disponible en soportedgis. salud.gob.mx/sis/.../pdf/SIS_CuestionarioFactorRiesgo2010.pdf

19. Organización Mundial de la Salud. Obesity: preventing and managing the global epidemic. Report of a WHO consultation on obesity. Report series. Ginebra: OMS, 2000.

20. International Society for the Advancement of Kin anthropometry (ISAK) girth/circumference measures [Recuperado el 13 de Septiembre de 2007]. Disponible en http:www.topendsports.com/testing/tests/girths.htm

21. Reglamento de la Ley General de Salud en materia de la investigación para la salud. Diario Oficial de la Federación. 7 de febrero de 1984. [Recuperado el 13 de Septiembre de 2007]. Disponible en http//www.seguro-popular.salud. gob.mx/descargas/marco_juridico/07_01_lgs.pdf.

22. Arenas G, Torres MA, Santillán M, Robles L. Factores de Riesgo para Enfermedades Crónicas en Universitarias del Área de la Salud. Enfermería Universitaria. 2008. 5(1): 22-6.

23. Bañuelos Y, Bañuelos P, García JC, Garvalena MJ, Ramírez MC, Alarcón MA. Obesidad y Alteración de la Glucosa en Ayuno en una Población Determinada. Desarrollo Científico de Enfermería. 2009; 17(3): 101-5.

24. Hu FB, Manson JE, Stampfer MJ, Colditz G, Liu S, Solomon CG, et al. Diet, lifestyle, and the risk of type 2 diabetes mellitus in women. N Engl J Med 2001; 345: 790-7.

25. Varo CJ, Martínez HM, Martínez GM. Beneficios de la actividad física y riesgos del sedentarismo. Medicina Clinica. 2003; 1(21): 665-72.

26. Mozaffarian D, Kamineni A, Carnethon M, Djousse L, Mukamal KJ, Siscovick D. Lifestyle Risk Factors and New-Onset Diabetes Mellitus in Older Adults. American Medical Association. 2009; 169(8): 798-807. 